\title{
Understanding Smart Wearable Sensors Technology: Impact on Human Health and Fitness
}

\author{
${ }^{* 1}$ IDOGA, EP; ${ }^{2}$ ADAMU, AI \\ ${ }^{I}$ Federal University Dutse, Cyber Security Department, Nigeria \\ ${ }^{2}$ Ibrahim Badamasi Babangida University Lapai, Computer Science Department, Nigeria \\ *Corresponding Author Email: dr.pat@fud.edu.ng; albattuta88@gmail.com; Tel: +2348166744812
}

\begin{abstract}
In the field of health care management, smart wearable devices and its supporting technologies have tremendously made a name all around the globe. Smart watches and other sensor trackers are practically being used by various people and its usage has shown to be accompanied with lots of benefits. This technology was envisaged to play a vital role in the healthcare needs of people; especially with applications in the healthcare sector. The objective of this study, therefore, is to evaluate the technological impact of wearable sensors in human health and fitness (HHF). A web based survey was used for data collection for the period of one month. Emails were sent to registered members of a particular gym who uses any of the smart wearable sensors in keeping fit. The study findings indicate that among the smart wearable devices examined, smart wristwatches $(45.6 \%)$ appears to be the most commonly used wearable sensor device followed by smart wrist bands $(34.7 \%)$, smart textiles $(10.7 \%)$ and smart rings $(9.1 \%)$. This signifies that a large number of people can effortlessly use SWSs and devices and are optimistic about its support in their daily healthcare/fitness needs. Users are positive on the technological prospects of SWSs and devices; although there is a gap between personal motivation to use wearable devices and trust in the confidentiality and privacy of data generated.
\end{abstract}

\section{DOI: https://dx.doi.org/10.4314/jasem.v24i7.19}

Copyright: Copyright $\left({ }^{\circ} 2020\right.$ Idoga and Adamu. This is an open access article distributed under the Creative Commons Attribution License (CCL), which permits unrestricted use, distribution, and reproduction in any medium, provided the original work is properly cited.

Dates: Received: 16 May 2020; Revised: 29 June 2020; Accepted: 07 July 2020

Keywords: Devices, Health, Fitness, Wearable, Sensors

The advent of affordable and efficient portable sensors is attributed to different factors, one of which is the introduction of wireless communication networks (Kwee-Meier et al., 2016; Lee et al., 2016). Sensors have the ability to dictate, process and transmit different signals, as a result, they are used by healthcare professionals to monitor the patient's health. The resultant effects which are, the development of smart wearable sensors, electronics or advanced technologies using computers (De-Acutis and De-Rossi, 2017). Smart wearable sensors (SWSs) refer to electronic tools that are translated into items that can be preprocessed into the body with enhanced computing devices that grant/process inputs (KweeMeier et al., 2016). SWSs could be used by attaching on clothing, patches in the skin, wristwatches and wearable glasses. However, recent studies have shown that SWSs can also be inserted into the body of a patient via surgical procedures (Chan et al., 2015). Another key feature of SWSs is its hands-free function that could enable users to monitor their health with relevant data while performing their daily routine (Woollaston, 2017; Kaewkannate and Kim, 2016). Some other characteristics include; access, comfort, portability, general multi-functions, importance, reliability of use, and practicability in real time. Consequently, according to Ritevski and Chen (2018), some SWSs such as the Insulin Monitors (INM) and Cardiac Event Monitors (CEM) has been provided freely by laws governing the healthcare body.

SWSs has been seen to be of great benefit to the wellbeing of the user, hence some are designed in the form of simple fitness tracker with tracking capabilities as well as accessing and monitoring user's physical activities which are in line with medical fitness and wellness of patients (Dohee et al., 2016; Lee et al., 2016). Significant parameters such as, the heart-beat rate, the blood pressure (BP), the pulse rate, muscle actions, sugar and glucose level, electrocardiogram (ECG), body temperature, digestion of oxygen saturation, the stress levels and eating habits are measurable by SWSs (Jayoung et al., 2018). Each of these parameters requires a certain type of sensor at a specific part of the human body. The goal of this study is to investigate the technological impact of SWSs in human health and fitness (HHF). Fitness wearable sensors (FWSs) are used in tracking and accessing personal health and daily routine checks. The potentials in SWSs has necessitated the massive 
boost in the technology sector, resulting in the production of fitness SWSs such as smart rings, wrist bands, wrist watches and smart textile sensors, etc. (Witte and Zarnekow, 2019). In this study, we shall consider the above named fitness SWSs.

Smart rings (SRs): Smart rings (SRs) are one of the many finger augmentation technologies. They are made of electronic contraption and sensors and mostly worn on the fingers. Other finger augmenting technologies include the numerical gloves and finger nails display. SRs has input/output competences in which they are able to detect motion, gestures, muscle activities and provide feedbacks (Gheran et al., 2018). Incorporating physical checks together with wearable technologies requires sensor placement. Hence, Asada and friends proposed the smart ring for the analysis of heart rate and blood oxygen saturation (Porciuncula et al., 2018).

Smart wrist bands (SWBs): Smart wrist bands (SWBs) consist of numerous sensors capable of sensing motion, temperature, pulse and other vital signs measurement. SWBs was first used on athletes to monitor their heart rate performance in real-time during an exercise routine (Koydemir and Ozcan, 2018).

Smart wrist watches (SWWs): Smart wrist watches (SWWs) are embedded with several sensors for keeping track of a user's fitness and health metrics as regards blood pressure, heart rate, calories burned, number of steps taken, blood glucose and sleep quality (Miller, 2019).

Smart textiles (STs): Smart textiles (STs) are used to monitor the physiological vitals of users. STs such as smart socks provide 3D measurement of pressure distribution of the user's whole foot while walking. This collects vital information of the user and enhance tailored fabrication of shoes for diabetic foot syndrome patients, thereby, reducing pain in the foot and subsequent foot ulcer (Koydemir and Ozcan, 2018).

Despite the many benefits such as remote monitoring, fitness tracker, CEM, INM etc. (Ritevski and Chen, 2018) associated with SWSs, some users still believe that the technological impact of these devices is negligible especially in HHF. Therefore, this study aims to evaluate the technological impact of SWSs in HHF.

\section{MATERIALS AND METHODS}

This is a closed, voluntarily web-based survey. As stated earlier, a web-based survey platform (WBSP) (https://www.surveymonkey.com) was used in questionnaire design and collection of data. The questionnaire was anonymous and protected by Norton and TRUSTe with approximately ten (10) minutes to complete. The WBSP clearly shows progress indicators and personal comments for any of the questions entered by the compilers. According to a "Checklist for Reporting Results of Internet ESurveys" (CHERRIES) (Masconi et al., 2019), all questions are compulsory. Based on this checklist, only completed questionnaires were examined exclusive of questionnaires with omitted data. While on the WBSP, it is possible to go back to a previous page through a "back button" in order to reverse answers before submitting. Answers were automatically collected on the Survey-Monkey database.

Questionnaire development: The questionnaire used in this study was adopted from the study of Mosconi et al., (2019) and administered through Survey Monkey - a web-based survey platform. Adequate caution was taken to ensure the questionnaire items consist of words such as frequency of use, mostly used, utilities, issues associated with use, as well as the technological impact in relation to healthcare technologies focusing on HHF wearable sensors. Prior to the distribution of the questionnaires to participants, a pretest of the questionnaire was presented to knowledgeable experts in the field of HHF and eHealth technologies: this is to ensure clarity, completeness and necessary suggestions. Their response/consensus resulted in the final questionnaire that was used in the study.

Participant's selection: Participants of the survey were registered members of a gym center in Nigeria $(\mathrm{N}=398)$ who are users of any of the SWSs mentioned above. The gym center was considered appropriate for the survey as a result of its relentless advocacy on the necessity of wearable devices for its members in keeping track of physical activities and maintaining a good fitness level; especially, for those with underlying chronic diseases (Pardamean et al., 2019). The survey URL link was sent through emails to the participants through their email addresses provided at the time of membership registration. Participants were asked to first, give their consent before proceeding with the survey. Additional care was taken to ensure that the duplicate and wrong email addresses as well as non-users of wearable sensors were eliminated $(\mathrm{n}=$ 23). A reminder email and follow-up call were also given to each of the participants two weeks later. The whole process of the survey took a month approximately (01 February - 02 March 2020). 
Data analysis: Statistical Analysis System (SAS version 9.4) was employed to carry out a descriptive analysis of collected data. By considering suggestions from an earlier study (Mosconi et al., 2019), pattern of answers by respondents were analyzed by their age and personal use of wearable sensors. Only results with substantial patterns in a set alpha level of 0.05 was considered for a $\mathrm{P}$ value evaluation.

\section{RESULTS AND DISCUSSION}

A total of 375 responses was collected, with a response rate of $94.22 \%(375 / 398)$. Data analysis was conducted on 375 correctly filled questionnaires since 23 of the responded data was insufficient to be considered in the analysis. The Table 1 summarizes the respondent's characteristics. Out of the 375 responders who participated in the survey, 110 (29.3\%) were male, while $265(70.7 \%)$ were female respectively. Indicating that, in terms of health care technological advancement, women are well knowledgeable and represented while the men are fairly represented. Respondents were aged 18-60 with the mean age of 39 . An appreciable level of technology use is clearly seen as respondent agreed to the usage of at least one SWS device; with SWWs $171(45.6 \%)$ been the most used wearable devices, followed closely by SWBs 130 (34.7\%). On the other hand, the SRs and STs seems to be unpopular and least desired with 34 $(9.1 \%)$ and $40(10.7 \%)$ respectively. In terms of the utility of SWSs, the majority of the respondents (58.4\%) reported that, these wearable sensors are not only useful in keeping track of their physical health, but also motivate them to better understand their healthcare needs. While $49.1 \%$ of the respondents believed that indeed, wearable sensors enhances the communication between them and their doctors, a lesser percentage of the respondents $(36.3 \%)$ are of the opinion that wearable sensors does not in any way reduce the cost of healthcare since the actual cost of health care is borne by the users. On the contrary, some of the respondents, finds wearable sensors not to be useful and of no effect (Table 2). According to the 375 respondents, among the wearable sensors examined, SWBs has the most significant positive impact in HHF and healthcare communications, SWWs also had a significant impact on HHF with 168 (44.8\%) responders and $159(44.2 \%)$ of inclinations respectively; these were trailed by the SRs and STs with $106(28.3 \%)$ and $82(21.9 \%)$ accordingly. However, some respondents opined that these wearable devices are associated with some negative influences while others are of no effect whatsoever (Table 3).

Table 1. Characteristics of responders $(\mathrm{N}=375)$

\begin{tabular}{ll}
\hline Characteristics & Frequency, n (\%) \\
\hline Sex & $110(29.3)$ \\
Male & $265(70.7)$ \\
Female & \\
Age & $257(68.5)$ \\
$\leq 49$ & $65(17.3)$ \\
-59 & $53(14.1)$ \\
$>60$ & \\
Personal use of wearable sensors & $34(9.1)$ \\
Smart rings & $130(34.7)$ \\
Smart wrist bands & $171(45.6)$ \\
Smart wrist watches & $40(10.7)$ \\
Smart textiles & \\
\hline
\end{tabular}

Table 2. Utility of healthcare apps and sensors in $\operatorname{HHF}(\mathrm{N}=375)$

\begin{tabular}{|c|c|c|c|}
\hline \multirow[t]{2}{*}{ Utility } & \multicolumn{3}{|l|}{ Responders, n (\%) } \\
\hline & Useful & Not useful & No effect \\
\hline To understand one's own health & $219(58.4)$ & $92(24.5)$ & $64(17.1)$ \\
\hline To improve patient-doctor communication & $184(49.1)$ & $168(44.8)$ & $23(6.1)$ \\
\hline To reduce cost of healthcare & $136(36.3)$ & $198(52.8)$ & $41(10.9)$ \\
\hline
\end{tabular}

Table 3. Technological impact of SWS on medical care and health

\begin{tabular}{llll}
\hline SWSs & Positive impact & Negative impact & No impact \\
\hline Smart rings & $106(28.3)$ & $206(54.9)$ & $63(16.8)$ \\
Smart wrist bands & $168(44.8)$ & $139(37.1)$ & $68(18.1)$ \\
Smart wrist watches & $159(42.4)$ & $165(44.0)$ & $51(13.6)$ \\
Smart textiles & $82(21.9)$ & $193(51.5)$ & $100(26.7)$ \\
\hline
\end{tabular}

Table 4. Issues associated with the use of SWS and devices

\begin{tabular}{llll}
\hline Issues & Yes, $\mathrm{n}(\%)$ & $\mathrm{No}, \mathrm{n}(\%)$ & I don't know, n (\%) \\
\hline Technical obstacles & $104(27.7)$ & $235(62.7)$ & $36(9.6)$ \\
Personal motivations, for example not being able to use them & $213(56.8)$ & $130(34.7)$ & $32(8.5)$ \\
Little trust in usefulness of data recorded & $120(32.0)$ & $198(52.8)$ & $57(15.2)$
\end{tabular}

IDOGA, EP; ADAMU, AI 
Low trust in confidentiality and privacy of data

Low trust in accuracy and reliability of data recorded

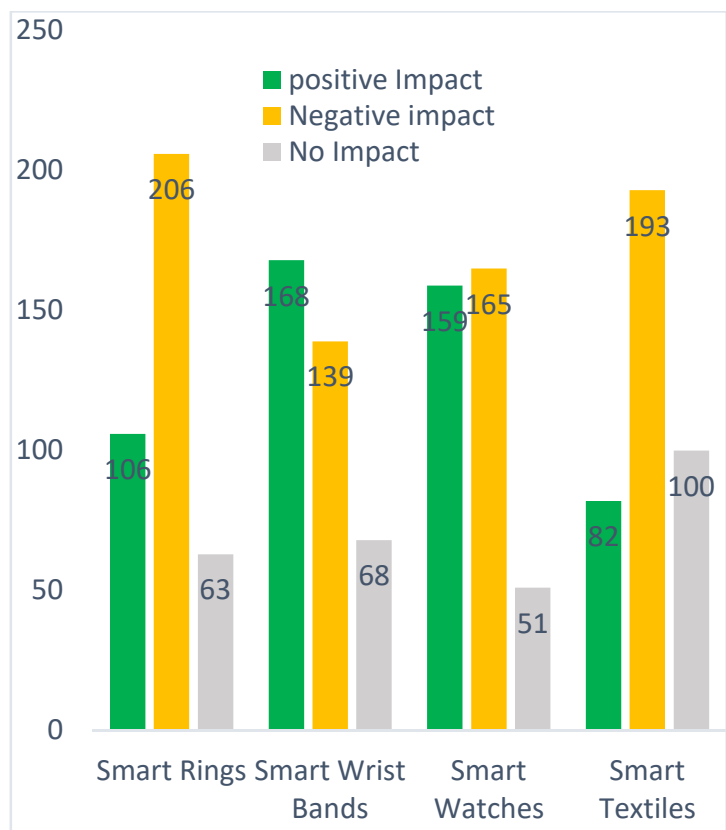

Fig 1: Technological impact of Smart Wearable Sensors

As common with every technology, respondents who participated in the survey reported some issues associated with their use of SWSs and devices. Among these issues is personal motivation: for instance, anxieties related to technical know-how, and unavailability of a smart phone along with associated devices; re-affirming the study of Mosconi et al., (2019), closely trailed by inadequate prove to substantiate their accuracy and consistency, usefulness of data as well as technical obstacles. Surprisingly, low trust in confidentiality and privacy of data which is envisaged to greatly hinder the adoption and utilization of SWSs turned out to have the least concern with only $90(24.0 \%)$ of the respondents affected. A small portion of respondents seems not to have any idea concerning the issues associated with SWSs and devices as can be seen in the table 4. This study on SWSs examined users' perspectives on the technological impact of SWSs in HHF. Findings from the study indicate that, among the SWS devices examined, SWWs appears to be the most commonly used wearable sensor device; providing access to healthcare information such as: physical activities, diet and weight management etc. followed by SWBs. Findings from the study is related to a recent survey on wearable health apps and wearable devices, where the most used healthcare apps were reported to be informative apps (Mosconi et al., 2019). In addition, this study is in contrast to those reported by (Wen et al., 2017) where bracelet-type wearable devices seems to be mostly preferred. Respondents are optimistic
$90(24.0) \quad 238(63.5) \quad 47(12.5)$

$128(34.1) \quad 220(58.7) \quad 27(7.2)$

about the future benefits of SWSs in HHF; however, there seems to be a gap between personal motivation to use SWSs and trust in the confidentiality and privacy of data. There is therefore a need to further enlighten users on the importance of continuous usage. More indication needs to be provided to substantiate the effectiveness of the devices since they are not even worried about the confidentiality of data. Concerning the accuracy and reliability of data, users seem not to be bothered about the data generated by these devices; this is very significant since the lack of this might hinder the adoption of wearable sensors and devices (BinDhim and Trevena, 2015). Nevertheless, designers must endeavor to design products bearing in mind, ease of use and easy interpretation of data is key to its acceptance and continuous usage. It is also crucial, complete product design trials are carried out on people to determine the actual needs of users and the accuracy of data generated. Studies (Covolo et al., 2017; Mosconi et al., 2019) has shown that these trials are limited due to some managerial constraints. The study has some limitations. In the first instance, the response rate $94.22 \%(375 / 398)$ may be too small for this kind of study as such, may not be adequate for generalization. Secondly, the respondents are all members of a particular gym center. It is likely that their point of view differs from those in other gym centers. Hence, it is necessary for a further study which will cover a wider inclusion of various types of users.

Conclusion: Varieties of SWSs abound; resulting in user's difficulty to determine the ideal SWSs device suitable to meet their healthcare needs. This study, which examined the technological impact of wearable sensors enhance the body of knowledge by identifying which of these devices user's find appealing and comfortable to use. Evidently there are issues associated with the use of SWSs; it is, however, expected that these issues be given the highest precedence to ensure a hitch free experience while using SWSs and devices.

Acknowledgements: The authors appreciate the management and staff of the establishment wherein the participants were recruited. Special appreciation also goes to all the participants who took part in the survey. This research was not funded.

\section{REFERENCES}

BinDhim, NF; Trevena, L (2015). Health-related Smartphone Apps: Regulations, Safety, Privacy and Quality. BMJ Innov. 1(2): 43-45. 
Chan, SS; Wong, DC; Cheung, YTD; Leung, DY; Lau, L; Lai, V; Lam, TH (2015). A Block Randomized Controlled Trial of a Brief Smoking Cessation Counselling and Advice through Short Message Service on Participants who joined the Quit to Win Contest in Hong Kong. Health edu. Res. 30(4): 609-621

Covolo, L; Ceretti, E; Moneda, M; Castaldi, S; Gelatti, U (2017). Does Evidence Support the use of Mobile Phone Apps as a Driver for Promoting Healthy Lifestyles from a Public Health Perspective? A Systematic Review of Randomized Control Trials. Patie. edu. and counse. 100(12): 2231-2243

De Acutis, A; De Rossi, D (2017). e-Garments: Future as "Second Skin"?. In Smart Textiles (pp. 383396). Springer, Cham.

Gheran, BF; Ungurean, OC; Vatavu, RD (2018). Toward Smart Rings as Assistive Devices for People with Motor Impairments: A Position Paper. In RoCHI. 99-106

Kaewkannate, K; Kim, S (2016). A Comparison of Wearable Fitness Devices. BMC Pub. Health. 16(1): 433

Dohee, K; Lee, S; Bahn, H (2016). An Adaptive Location Detection Scheme for Energy-efficiency of Smartphones. Perv. and Mobile Comput. 31, 6778.

Jayoung, K; Campbell, AS; Wang, J (2018). Wearable Non-invasive Epidermal Glucose Sensors: A Review. Talanta, 177, 163-170.

Koydemir, HC; Ozcan, A (2018). Wearable and Implantable Sensors for Biomedical Applications. Annu. Rev. of Analyt. Chem. 11, 127146.

Kwee-Meier, ST; Bützler, JE; Schlick, C (2016). Development and Validation of a Technology Acceptance Model for Safety-enhancing, Wearable Locating Systems. Behav. \& Inform. Tech. 35(5): 394-409.

Lee, J; Dohee, K; Ryoo, HY; Shin, BS (2016). Sustainable Wearables: Wearable Technology for Enhancing the Quality of Human life. Sustainability, $8(5): 466$
Miller, TW (2019). Smart Advances in Healthcare for Geriatric Patients." Am J Boimed. Sci \& Res - 1(3): 130-131. Doi:10.34297/AJBSR.2019.01.000526.

Mosconi, P; Radrezza, S; Lettieri, E; Santoro, E (2019). Use of Health Apps and Wearable Devices: Survey among Italian Associations for Patient Advocacy. JMIR mHealth and uHealth, 7(1): e10242.

Pardamean, B; Soeparno, H; Mahesworo, B; Budiarto, A; Baurley, J (2019). Comparing the Accuracy of Multiple Commercial Wearable Devices: A Method. Procedia Computer Science, 157, 567572.

Porciuncula, F; Roto, AV; Kumar, D; Davis, I; Roy, S; Walsh, CJ; Awad, LN (2018). Wearable Movement Sensors for Rehabilitation: a Focused Review of Technological and Clinical Advances. $P M \& R, 10(9)$ : S220-S232.

Ristevski, B; Chen, M (2018). Big Data Analytics in Medicine and Healthcare. J. of Integrat. Bioinforma. $\quad$ 15(3): 20170030. doi: https://doi.org/10.1515/jib-2017-0030

Wen, D; Zhang, X; Lei, J (2017). Consumers' Perceived Attitudes to Wearable Devices in Health Monitoring in China: A Survey Study. Comp. Meth. and progr. in Biomed. 140, 131-137.

Witte, AK; Zarnekow, R (2019, January). Transforming Personal Healthcare through Technology-A Systematic Literature Review of Wearable Sensors for Medical Application. In Proceedings of the 52nd Hawaii International Conference on System Sciences.

Woollaston, V (2017). These hearing aids link to smart TVs, doorbells and smoke alarms so wearers never miss a beat [Online]. Available: https://www.wired.co.uk/article/oticon-smarthearingaid\#: : text=These $\% 20$ hearing $\% 20$ aids $\% 2$ 0link $\% 20$ to, wearers $\% 20$ never $\% 20$ miss $\% 20 a \% 20$ beat\&text $=$ Ahead $\% 20$ of $\% 20$ this $\% 20$ year's $\% 20 \mathrm{Co}$ nsumer,link\%20directly\%20to\%20IoT\%20device s[Accessed 02 May 2020]. 